legislation has effected. The fact that there has been more industrial legislation in Great Britain during recent years than ever before in our history, and that our industries, instead of being better, are worse now than before, is in itself a proof that investigation is urgently needed. "The acquisition of data is the first necessity." From the study of these data there must gradually emerge principles of guidance in legislative matters of which we are in sore need : no greater benefit can be conferred by science on humanity at large than the discovery of these principles. It can scarcely be hoped that legislation will be on right lines in the absence of this guidance, which science alone can provide.

Let it not be forgotten that our civilisation (of which industry is the main support) has been built up by our forefathers and handed on to us as a precious heritage, and it is our first duty to posterity to see that it does not suffer while under our charge; but is passed on to those who will come after us in a condition somewhat better than that in which we received it; to do less than this we should be guilty of breach of a great trust. We are apt to boast of the great advance made by science, but what will be the verdict of the historian of the far future when, in recounting the history of the critical times through which we are now passing, and in view of later disasters, he makes the astonishing discovery that our legislation was framed without scientific guidance of any kind; that while it has occupied itself with the changes and movements taking place in stars and nebulæ millions of light-years away, which can affect our civilisation not at all, it neglected those taking place on our doorsteps on which our very existence depended ?

Meir,

Alan Blair.

Stoke-on-Trent.

May 4.

\section{Essential Structural Discontinuities in Certain Adsorbed Films}

IN connexion with some recent experimental work, it has been necessary to consider in detail the process of building up an adsorbed film which is formed by the adsorption on neighbouring solid atoms of the two atoms of a diatomic gas molecule, and in which the adsorbed atoms are immobile and stable. Such a film is necessarily imperfect and incomplete, having gaps or holes in it.

For the sake of definiteness, let us consider adsorption on a plane in which each surface atom is surrounded by four equally spaced other atoms. As the film is gradually built up, certain single surface atoms will find themselves surrounded by four filled places. Such atoms will be able to take no part in the adsorption process and will remain bare. The complete film thus of necessity has a sort of irregular mosaic structure.

It is important to know what proportion of the surface atoms remain bare. To do this an experimental numerical test has been carried out in which neighbouring pairs of points on a diagram of such a plane have been selected at random and occupied. It was found that about eight per cent of the total number of surface atoms remain uncovered. In order to test whether the particular assumption about the atomic arrangement is of importance, a similar test was carried out on a hexagonal arrangement, so that there could be six possible places for the second atom of a molecule to occupy. The numerical result was practically identical.

The actual case under consideration was that of oxygen on tungsten, and experimental evidence for the existence of these gaps has been obtained. The uncovered surface atoms will undoubtedly exert a much greater attraction on impinging oxygen molecules than the other parts of the surface. An adsorbed film of molecular oxygen has been found of the right amount to correspond to adsorption on these gaps in the atomic film.

For the adsorption of molecular oxygen, these gaps must be regarded as active parts of the surface. They would probably also be centres of catalytic activity if one were using a surface from which the atomic film had not been removed. If they were, an amount of oxygen small compared with that required to cover the surface completely would be sufficient to poison it.

A full account of experimental methods and their application to the study of these films is ready for publication, together with a discussion of other properties of the gaps.

In conclusion, it may be pointed out that almost any immobile adsorbed film, except one formed by the adsorption of gas molecules on neighbouring surface atoms without dissociation, will have essential irregularities of a similar type.

Department of Colloid Science,

\section{J. K. Roberts.}

Cambridge.

May 29.

\section{Lattice Distortion in Nickel-Iron}

RECENT investigations of W. A. Wood ${ }^{1}$ on the broadening of high-order Debye-Scherrer lines of copper and $\alpha$-brass have shown that the lattice distortion may be accompanied by changes in the spacings of various lattice-planes, pointing to a change in shape and (perhaps) size of the unit cell. The occurrence of such an effect can be very clearly shown for nickel-iron. For an alloy with 53 atomic per cent $\mathrm{Fe}$, the side-length of the unit-cube $a$ is $3.58 \mathrm{~A}$., so that the (400)-reflections with cobalt $K \alpha_{1}$ and $K \alpha_{2}$ radiation oceur at about $85^{\circ}$ and $87^{\circ}$

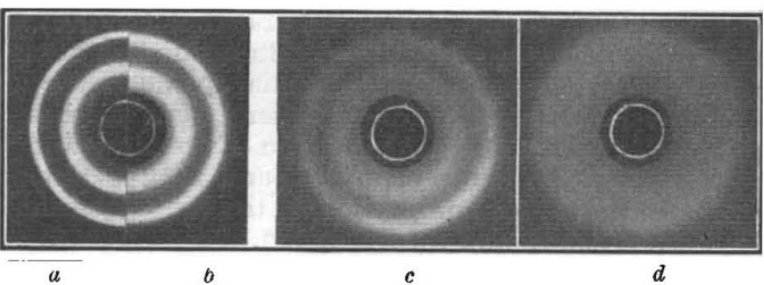

FrG. 1. Nickel-iron (400)-reflection, cobalt $K a_{1} \alpha_{2}$ doublet.

$a$, annealed band.

$b$, after 10 per cent compression perpendicular to plane of rolling.

$b$, after ${ }_{2}$ per cent compression perpendicular to plane of

respectively, and thus show extremely large dispersion (the distance of the $\alpha_{1} \alpha_{2}$-lines for a specimen to film distance of $12 \mathrm{~cm}$. being about $7 \mathrm{~mm}$.). Back-reflection photographs of discs, cut from recrystallised cold-rolled band, show these reflections with great intensity, as the recrystallisation texture is a very pronounced 'cube-texture' ('Würfellage'), all crystals lying with a cube-face and -side within $\pm 10^{\circ}$ parallel to the plane and direction of rolling ${ }^{2}$. The effect of compression-deformation is shown in Fig. 1, 